\title{
Affect and Place Representation in Immersive Media: The Parragirls Past, Present project
}

\author{
Volker Kuchelmeister \\ UNSW Sydney, Art and Design \\ Greens Rd, Paddington, NSW, 2021 \\ Australia \\ kuchel@unsw.edu.au
}

\author{
Lily Hibberd \\ UNSW Sydney, Art and Design \\ Greens Rd, Paddington, NSW, 2021 \\ Australia \\ I.hibberd@unsw.edu.au
}

\author{
Alex Davies \\ UNSW Sydney, Art and Design \\ Greens Rd, Paddington, NSW, 2021 \\ Australia \\ alex.davies@unsw.edu.au
}

\begin{abstract}
The emergence of a genre of virtual reality at the nexus of human rights is arguably transforming the documentary testimonial genre into one of an affective experience. This is a field of research that raises fundamental questions on the relation between sensation, testimony, evidence and memory. This paper outlines an investigation into the affective potential of immersive media and visualisation technologies to produce appropriate subjectivities for difficult memory and traumatic experience. The 3D immersive project under discussion presents a digital reconstructed reality of the former Australian punitive child welfare institution, Parramatta Girls Home. In this paper, we delineate how we have used the unique aesthetic properties of ambisonic sound, point-cloud representation and scenes of photographic veracity, and how the apposition of these formats provides an encounter with memory itself. Five former residents of Parramatta Girls Home direct our path through a complex visual and ambisonic acoustic environment, a combination we suggest empowers these women to attest to the embodied experience of contested memory.
\end{abstract}

Immersion. Empathy. Narrative. Memory. Imaging and video. Modelling. Point cloud. Multimedia.

\section{INTRODUCTION}

The 3D immersive narrative experience Parragirls Past, Present: Unlocking memories of institutional 'care', commissioned for UNSW EPICentre by The Big Anxiety, a festival of art, science and people held in 2017 in Sydney, offers a unique perspective on Parramatta Girls Home, a former statecontrolled child welfare institution situated adjacent to the convict-era Parramatta Female Factory in western Sydney, Australia. Up until the early 1980s, 'children at risk' were held in this place and subjected to unwarranted punishment, neglect and abuse, as evidenced in the Australian government royal commission into institutional responses to child sexual abuse (Commonwealth of Australia 2015). The project offers a novel configuration of reconstructed reality in a testimony-based narrative combined with an abstract representational format. Photographic veracity is suspended in an uncertain and dissolute world of seemingly unhinged temporalities. With one trend in CGI to generate ever more realistic depictions, this project takes a deliberate step away to deploy a more abstract reconstructed reality, the point-cloud representation. The intention of this research is to better understand how experimental approaches to veracity might be compatible with human memory. Memory encompasses a wide array of research disciplines across cognitive science, philosophy, trauma studies, history, heritage and film studies, among others. In this paper, we are concerned with examining the results of a recent collaboration with five former residents of Parramatta Girls Home, women who had previously collaborated as part of a collective of former residents called Parragirls Memory Project (PFFP Memory Project 2017). These five women - who were institutionalised at separate times through the 1960s and 1970s as teenage girls - provided us with the underpinning problematic of how to appropriately represent the relationship of difficult memory in the present and its impact on lived experience. This paper provides a detailed account of the conceptual, design and technical processes involved in creating this novel approach to the immersive media representation of traumatic memory and heritage place.

\section{REPRESENTATION OF MEMORY \& TRAUMA}

Witnessing trauma entails complex operations of memory. Dominant notions of truth and testimony do not however always empower who testify to 
trauma. These contradictions emerged at the outset of our collaboration with Parragirls on this project. The women highlighted two primary dilemmas of witnessing institutional experience. The first relates to the impossibility of absolute memory, lack of public belief and the burden of proof in judicial testimony. The second issue centres on the role of place in recollection, specifically the retrieval of traumatic memories embedded both in the present at the site and in the distant past. The women aptly define their return to Parramatta Girls Home fifty years later as akin to Alice going down the rabbit hole into wonderland.

In Parragirls Past, Present, it was necessary to consider the role of what is known in cognitive psychology as episodic memory as it relates to events and experiences that have happened in particular places at particular times. Tulving (2002) states that episodic memory 'makes possible mental time travel through subjective time, from the present to the past, thus allowing one to reexperience one's own previous experiences.' But we also know that in remembering past events we do not retrieve exact replicas, it is a constructive rather than a reproductive process (Bartlett 1932). Schacter (2007) argues that, 'memory is not a literal reproduction of the past, but rather a constructive process in which bits and pieces of information from various sources are pulled together'. The Parragirls project thus required a novel approach in order to represent both the veracity of the women's testimony and the specific difficulty of an unbroken recollection through the lens of time, dramatic changes to the site over fifty years and the impact of traumatic experience on remembering.

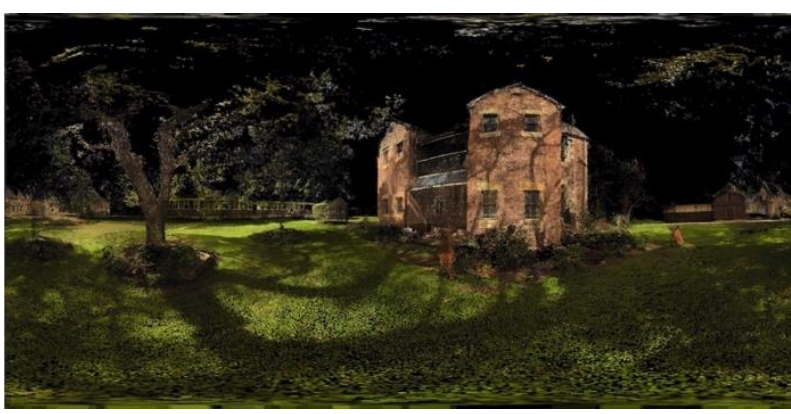

Figure 1: Parramatta Girls Home point-cloud representation in $360^{\circ}$ spherical format.

The decision to utilise photogrammetry to generate a point-cloud representation as a visual setting for this project is grounded on the mnemonic process involved in piecing together bits and pieces, while the fragmented aesthetic represents the contested nature of witnessing, trauma and long-standing public denial of abuse that took place in the Girls Home. The acoustic scape of the work likewise echoes the contested nature of visual representation of reality. Its layered temporal and multi-dimensional configuration and at times cacophonous arrangement of the women's narrations belies the apparent linear progression through the project's spaces.

\section{REPRESENTATION OF PLACE}

Remote sensing with LIDAR or Photogrammetry technologies and subsequent digital reconstruction and visualisation in point-cloud format is an established tool in engineering, mapping and cartography, archaeology, architecture and urban planning, geology and many other disciplines. Due to its precision, this technology is ideally suited to generate objective spatial datasets. Corner (2016) observes, 'Moving within a point-cloud model creates an entirely different reading of site, adding relationship and a value between things that are of a completely different order'. Corner (2016) further explores the idea of perceptual relativity and its difference from conventional perspectival imaging. Point-cloud models afford the possibility of an unlimited number of instant perspectives, unlike established perspectival models of perception. Girot (2014) notes that 'a pixel standing next to another... can actually hold an entirely different position that is distant several 100 meters in geographic computational reality'. This is one of the unique properties of a spatial point-cloud representation: a large number of individual elements are perceived to manifest surfaces or skins but those porous and distant points are only partially occluded and keep forming surfaces. It has an $\mathrm{x}$-ray like appearance, but with a very distinct aesthetic.

Point-cloud technology has also entered the entertainment industry, notably in the sciencefiction genre, where holographic representations are often depicted as a point-cloud visualisation. A recent example is Ridley Scott's Prometheus (2012), in which a spaceship interior is augmented by a holographic recording of events from a distant past. In this scene, the imperfect and fragmented point-cloud representation can be read as a metaphor for distant memories being reconstructed in the present. In the BBC TV production, Italy's Invisible Cities (BBC 2016), 3D scanning technology is employed to uncover the hidden history of three Italian cities. By visualising pointcloud datasets with colour information, the depictions in this television program achieve an extraordinary visual quality. The computergenerated scenes produce believable natural lighting conditions and build on the perception of authenticity and scientific accuracy associated with 3D scanning.

With spatial scanning technology becoming more accessible, smaller studios and independent artists 
have started to explore and manipulate point-cloud representations in imaginative and novel ways. ScanLab, a creative studio in the UK, explores 3D scanning technology in architecture and the creative industries, and sets benchmarks in regards to the quality and versatility of this technology. Another interesting example is In the Eyes of the Animal (2015), produced for VR. This artistic interpretation affords an embodied experience of animals in their natural habitat. The creators employ point-cloud visualisation in combination with particle animations, expanding an inherently static scene into the time domain. The project Palimpsest (2016) takes motion in point-cloud visualisation one step further by combining static point-cloud scenes with volumetric 'video'. This VR film contains recordings of personal stories and local histories of an urban space that is undergoing change. It captures the collective memory of a community and place at a point in time where those memories threaten to be displaced by development. This is a relevant example of the technology being employed in the narrative and documentary film domain.

For Parragirls Past, Present (2017) we focused on immersing the audience in an audio-visual narrative. The digital point-cloud reconstruction of the Parramatta Girls Home site (Figure 1) forms a visually evocative setting for a highly considered layering of stories that shift between present recollection and displacements of time and space, and attempts to bridge these two perceptual dimensions. We were distinctly aware of the advantages of point-cloud representations to simultaneously generate the perception of authenticity and scientific accuracy while presenting a fragmented and broken world. We deployed these dualities in a process that blurred the sense of reality into visual abstraction. This transcendence of prevalent distinctions between the real and the virtual is an integral part of our project. It demonstrates that abstraction influences the perception and understanding of affective encounters with documentary material, as well as the emotional response to place and testimonial forums of memory in immersive media environments. The following elaborates the technical and conceptual evolution of this project.

\subsection{Point-cloud format}

Point-cloud data is comprised of a large number of discreet elements, each with positional and optional colour information. This is also referred to as $X Y Z R G B$ point-cloud. Where $X Y Z$ are the Cartesian coordinates and RGB a colour value. This data does not contain a reference to the realworld scale or the spatial expansion of the pointcloud model. A scale factor is required to map the point-cloud data to a correct scale in representation. To draw a point-cloud on screen, the software ingests all elements, distributes them according to their positional information and assigns the colour value. The size and shape of each individual element is variable. For a large dataset $(<10 \mathrm{M})$, simple rectangles facing the camera are a lot faster to draw then primitive spheres.

\subsection{Survey and data acquisition}

Two different methods were employed to capture the datasets at the site of Parramatta Girls Home: photogrammetry for 3D data in the form of pointclouds and textured mesh models, and photography with panoramic stitching for $360 \times 180^{\circ}$ stereoscopic panoramas. Photogrammetry is based on making measurements from photographs, especially for recovering the exact positions of surface points in a scene. With feature detection and correlation amongst multiple photographs, from various points of view, a photogrammetry algorithm estimates the camera intrinsic and extrinsic parameters and, by doing so, generates a 3D model of a scene or object. For this project, we experimented with a terrestrial and an aerial survey.

\subsubsection{Terrestrial survey}

The terrestrial survey was conducted with a custom designed, low-cost four-camera rig, where the four compact action cameras were mounted on a bicycle helmet, pointing in four directions (Figure 2). The cameras operated in time-lapse mode, taking photographs every 2 seconds, while the surveyor slowly walked around the site on tracks pre-defined by Parragirls collaborators (Figure 3 ).
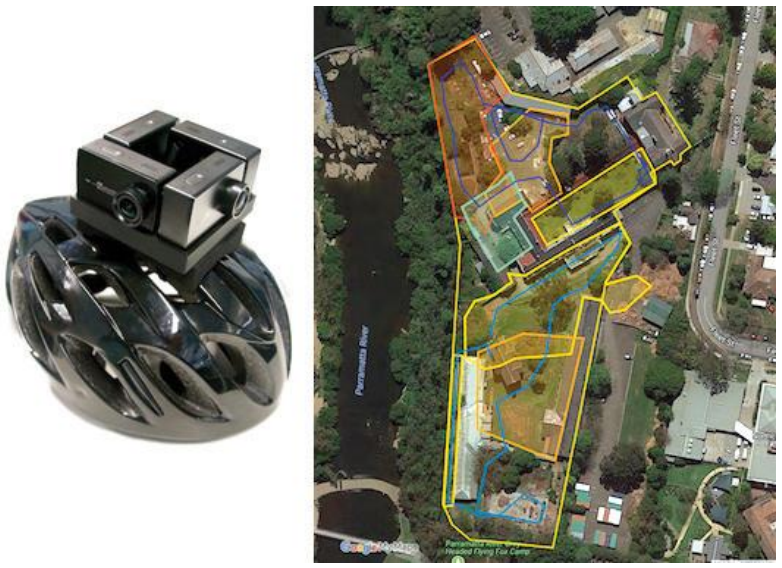

Figure 2, 3: Custom low-cost camera rig for terrestrial site survey (I). The site in Parramatta, 1.5ha. The tracks and survey segments are highlighted (r).

This particular camera model ( $Y i \quad 4 k$ ) has a photographic resolution of $4000 \times 3000$ pixel and an ultra-wide lens $\left(2.6 \mathrm{~mm}\right.$ focal length or $150^{\circ}$ field of view). Subjectively, the image quality is not stellar, the focus is soft and JPEG compression artefacts 
are present, but this is out-weighed by its low-cost and the simplicity of set-up during surveying. By capturing a large number of partly redundant images, it is tolerable for some of the photographs to be discarded by the photogrammetry algorithm. For the 1.5ha site, the terrestrial survey resulted in 3200 photographs from the four cameras, split into multiple segments for processing (Figure 3).

\subsubsection{Aerial survey}

Aerial surveys are traditionally utilised in cartography to generate accurate relief maps. For our project, we wanted to investigate if the accuracy and quality of the resulting dataset could improve upon the terrestrial survey. For this a camera drone (Dji Phantom 3) was preprogrammed to survey the site in a regular strip pattern, at a height of $50 \mathrm{~m}$ to avoid collisions with tall trees. The camera was pointing straight down and taking photographs at a two seconds interval. A total of 275 images were captured, airtime was $\sim 10 \mathrm{~min}$ (Figure 4).

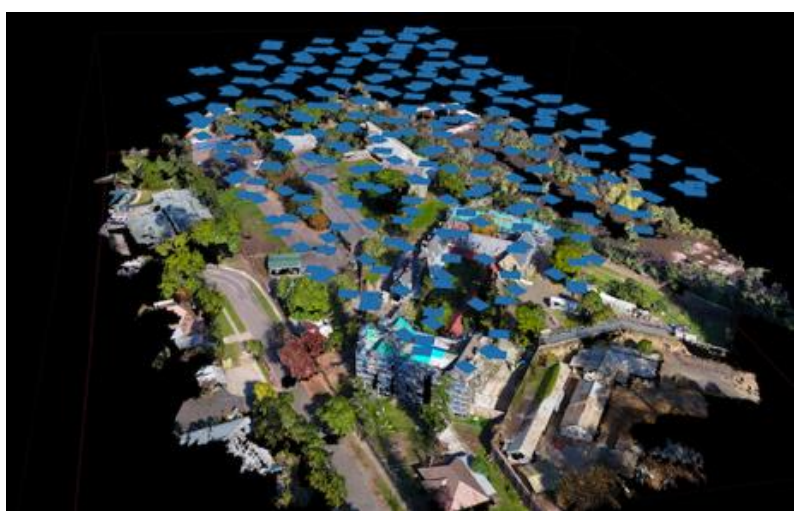

Figure 4: Aerial survey Parramatta site. After photogrammetrical processing (Agisoft Photoscan).

It became apparent after processing and visually evaluating the dataset that the aerial perspective is not very well suited to a representation in human scale and from a grounded perspective. The general topography and rooftops are represented in high detail, whereas building facades, sections of ground are partially occluded by trees, and in general a low level of detail on the ground plane was not suitable for our application (Figure 5).

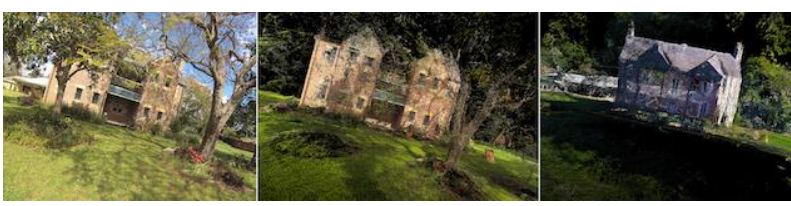

Figure 5: Comparison level of detail: photograph (I), terrestrial $(m)$ aerial $(r)$ reconstruction from a corresponding point of view.

This issue could be partially overcome by repeating the survey while the camera is tilted more towards the horizontal plane. By doing so it would capture more of the vertical detail, but not to a point where it would compete with the terrestrial approach. Ideally one would merge the terrestrial with the aerial dataset, but we were satisfied with the quality and the look of the existing terrestrial point-cloud model and discarded the aerial survey.

\subsubsection{Interior spaces}

Selected interior spaces were captured separately, using the same four-camera rig and alternatively with a DSLR camera, resulting in a total of $\sim 6800$ photographs. After evaluating the action camera photographs, it became clear the quality was not adequate to generate reasonably high-resolution textures for 3D mesh representation (Figure 6). This was due to relatively low light and consequent motion-blur in the imagery because of the mobile survey technique. The DSLR camera was thus employed to take higher quality photographs for photogrammetrical processing. In our application, the fidelity of the texture, more than the accuracy of the mesh, defines the quality and the look of the 3D model in representation (Figure 7,17 ). For a 25 sqm room, approximately 150-200 images were sufficient to generate a $3 D$ mesh with highresolution textures.

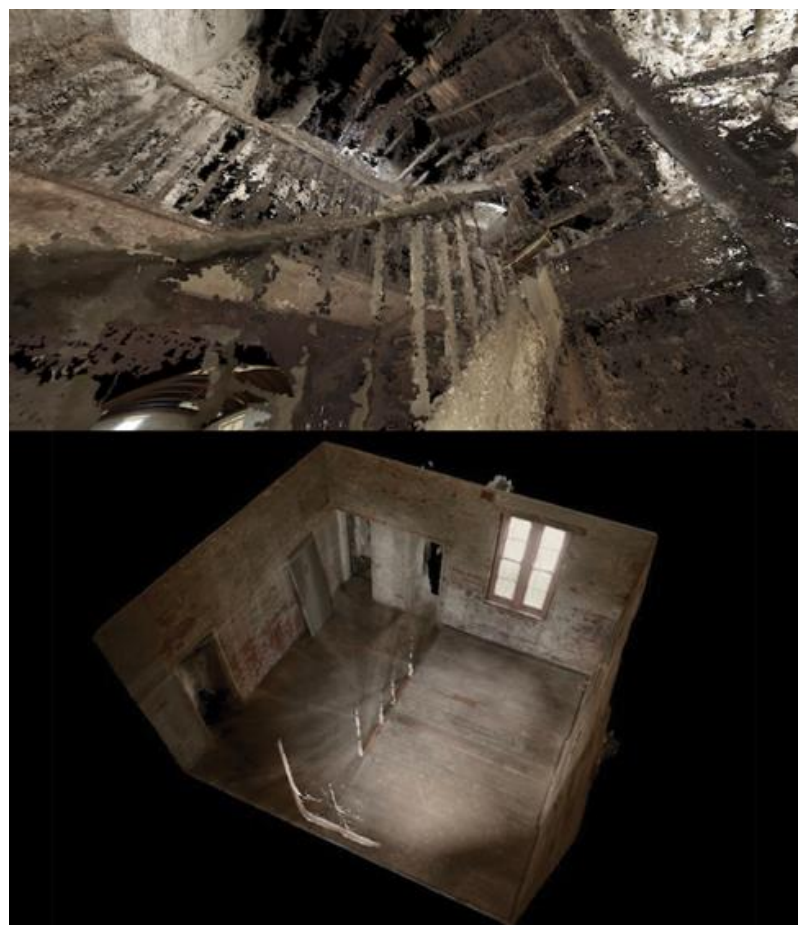

Figure 6, 7: Point-cloud representation, captured with action camera rig $(t)$. Textured mesh of interior spaces, captured with a DSLR (b).

As an alternative to 3D point-cloud and mesh representations, we captured stereoscopic $360 \times 180^{\circ}$ panoramic photographs of selected interior spaces (Figure 18). The final film features four interior spaces in photographic representation. The contrast between the digitally reconstructed reality and the comparative photographic realism 
breaks up the visual flow in the film. These 3D panoramic photographs were captured with a single DSLR camera in portrait orientation with a wide $(15 \mathrm{~mm})$ lens on a tripod with motioncontrolled head. We took 36 overlapping photographs for the horizontal panorama, one set for the left and one for the right eye view, plus two additional rows to cover the entire spherical projection. The photographs were then colour processed, stereoscopically stitched (PTGui) and rendered as $16 \times 16 \mathrm{k}$ top/bottom 3D equirectangular image. Great care must be taken to ensure the imagery has correct stereoscopic depth around the horizontal plane, as traditional stitching is not very well suited for the off-axis photographs needed to achieve depth disparity.

\subsection{Data processing and filtering}

The photographic datasets for the point-cloud reconstruction were processed with a commercial photogrammetry application (Agisoft Photoscan). The workflow is comprised of: 1) the automatic alignment of source images (feature extraction) which results in a sparse cloud, 2) the refinement to a dense point-cloud (Figure 8) the optional generation of a 3D mesh model and the creation of UV textures. Throughout these steps adjustment in accuracy, quality and filtering can be manipulated. The processing time for the six datasets with a total of $\sim 3200$ action camera images was approximately 5 days on an iMac (Intel Core i5 with integrated GPU).

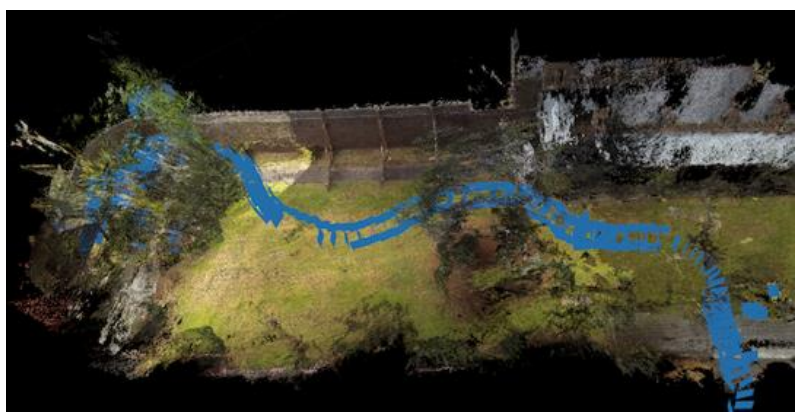

Figure 8: Photogrammetry processing terrestrial survey. The photographs of the camera cluster are represented as blue quads (Agisoft Photoscan).

After processing, the raw XYZRGB point-cloud datasets were exported for further processing in an application based on the open Point-Cloud-Library (Cloud Compare). The workflow includes the cropping, scale and translation, the application of a SOR (Statistical Outlier Removal) filter, spatial subsampling to achieve a uniform density amongst multiple datasets and the registration of the multiple datasets, so only one point-cloud model contains the entire 1.5 ha site.

The final terrestrial dataset for the 1.5ha site is comprised of $24 \mathrm{M}$ points (Figure 13). The aerial survey was straightforward to process: no segmentation of the site was needed, and the final dataset, encompassing 3ha, was exported unfiltered with a total of 55M points (Figure 4).

\section{VIEWING PLATFORM}

The project delivery platforms are a commodity VR headset (the target is Gear VR) and the large-scale cylindrical immersive environment EpiCylinder (Figure 9, 12) at UNSW Sydney Expanded Perception and Visualisation Interaction Centre, to date, the world's highest resolution immersive 3D cinema. EpiCylinder provides a unique delivery platform for ultra-high resolution digital content at a real-world scale for up to 15 viewers at a time.

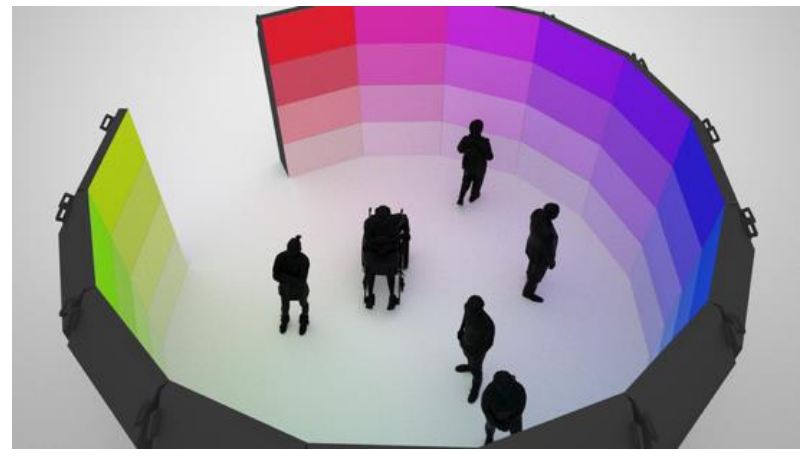

Figure 9: EpiCylinder at the UNSW's Expanded Perception and Visualisation Interaction Centre. A cylindrical stereoscopic immersive environment. Radius: $3.2 m$, height: $2.98 m$. Resolution: $26880 \times 4320$ pixel.

Its physical dimensions are, $3.2 \mathrm{~m}$ diameter, 340으, $2.98 \mathrm{~m}$ height, with a vertical field of view of $50^{\circ}$. It consists of four rows of fourteen slim-bezel DLP rear-projection display cubes (EyeVis EC-SLIM) each in full HD, which operate in $120 \mathrm{~Hz}$ active stereoscopic 3D. This amounts to a total resolution of $26880 \times 4320$ pixel. The sound system is designed to generate and play spatialised ambisonic sound on two rows of 16 speakers plus a sub-woofer.

Table 1: Comparison delivery platforms

\begin{tabular}{|l|l|l|l|l|}
\hline & $\begin{array}{l}\text { Field of } \\
\text { view }\end{array}$ & Resolution & Audio & $\begin{array}{l}\text { Viewing } \\
\text { experience }\end{array}$ \\
\hline $\begin{array}{l}\text { EpiCyli } \\
\text { nder }\end{array}$ & $\begin{array}{l}340 \times 50 \cong \\
\text { cylindrical }\end{array}$ & $\begin{array}{l}26 \mathrm{k} \\
\text { stereo 3D }\end{array}$ & $\begin{array}{l}\text { Ambisonic } \\
\text { 32 chan. }\end{array}$ & $\begin{array}{l}\text { Social, up to } \\
15 \text { viewers }\end{array}$ \\
\hline VR & $\begin{array}{l}360 \times 180^{\circ} \\
\text { spherical }\end{array}$ & $\begin{array}{l}4 \mathrm{k} \\
\text { stereo 3D }\end{array}$ & 2 chan.HRT & $\begin{array}{l}\text { Intimate and } \\
\text { personal }\end{array}$ \\
\hline
\end{tabular}

\section{CINEMATIC REPRESENTATION}

The project was realised as a linear experience without any viewer driven interactivity. While an interactive approach might have arguably generated a heightened sense of presence in a VR viewing context (Schuemie 1999), in dialogue with 
the five Parragirls it became evident that allowing viewers freedom to 'explore' the institutional spaces was incompatible with their lack of freedom in those spaces of confinement. A linear mode was thus elected to allow us to drive the narrative flow, which would also to convey to viewers the experience of being subject to institutional control.

Cinematic conventions only partly apply when working outside the framed image in a $360^{\circ}$ space. Traditional montage, that enables a viewer to reconstruct the architecture of a scene, was replaced here by a series of slow tracking shots with a virtual $360^{\circ}$ camera, shot from a low point-ofview, intended to be that of a child. To resolve the transition from a tracking shot within the exterior point-cloud model to a static depiction of an interior space, the camera 'breaks' through the point-cloud skin, as if the exterior skin is stretched to then 'break', while the scene dissolves to either a photographic panoramic representation or a realistic textured 3D mesh model (Figure 17, 18). The film has a total of 24 shots with a final duration of 23:05 minutes.

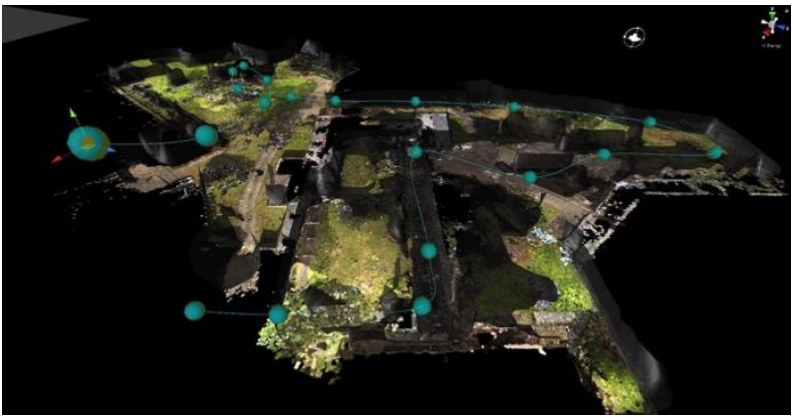

Figure 10: Path of the virtual 360 camera within the point-cloud scene.

With the aesthetics of the point-cloud representation being central to the project, we ran a series of experiments to adjust various visual attributes. Working with custom shaders in a $3 D$ game engine (Unity $3 D$ ) to render the points, we were able to perform adjustments in real-time while reviewing the scene in EpiCylinder and on a VR headset. We focused on a combination of five parameters: point shape, point size, point size relative to viewer/camera distance, point transparency and gamma luminance.

The relationship of space and time was critical, since setting the pace of the moving camera establishes a cinematic flow. Setting this tempo is a creative choice but also plays an important role in the viewer's level of comfort within an immersive platform. In this work, the viewer cannot control locomotion, only the direction of gaze, so motion sickness induced by the visual-vestibular conflict is a real possibility. Referring to So et al. (2001) and our own observations in EpiCylinder, we established a conservative very slow pace $(\sim 2$ $\mathrm{km} / \mathrm{h}$ ), significantly slower than average human walking speed $(\sim 5 \mathrm{~km} / \mathrm{h})$ and the speeds tested in So's study. There is also generally no acceleration in the camera motion. On a cinematic level, this slow pace is well suited for a narrative-driven project, especially one that presenting confronting material and trauma testimony. It gives the viewer 'breathing space' to comprehend and reflect on what they are hearing.

\section{RENDERING, COMPOSITING AND PLAYBACK}

While we were able to render the final $24 \mathrm{M}$ element point-cloud in near real-time ( 20fps) and full resolution with the EpiCylinder computer cluster, we pre-rendered all sequences and employed traditional editing and compositing. This approach simplified the integration of the interior photographic panoramas with the computergenerated visuals and allows for colour correction for a particular platform.

The main challenge was with the ultra-high resolution of the EpiCylinder target platform. We tested a series of still images of the point-cloud representation in increasing resolution and settled on working with $16 \mathrm{k}$ (16,384 pixel) horizontal. At lower resolutions the single points appeared slightly blurred.

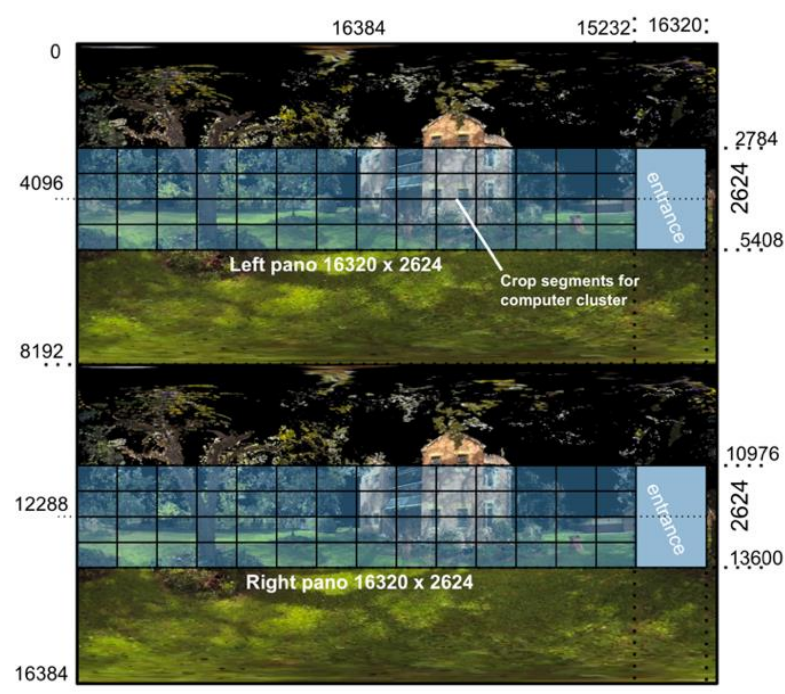

Figure 11: Render resolutions, slicing and formatting the imagery for EpiCylinder display.

With the final film duration being $\sim 23$ minutes, rendering over 41 thousand frames (at $30 \mathrm{fps}$ ), each $16 \times 16 \mathrm{k}\left(360 \times 180^{\circ}\right.$ equirectangular format in stereoscopic 3D), placed considerable challenges on the render pipeline. The render-engine was based on Unity 3D executable scenes with a custom shader for the point-cloud model and a virtual camera cluster solution for the stereoscopic 
$360 \times 180^{\circ}$ format. The camera cluster operates in cubic format (8x8k per cube) and a mesh to warp the cube sides to equirectangular format. After compositing and slicing for the EPICylinder display cubes (Figure 11), the sequences were encoded in Hap-Q for playback. For VR viewing, we exported a 4k (3840x2160 pixel) 3D equirectangular H265 encoded version. Close to the end of production, a total of $100 \mathrm{k}$ frames were rendered (including outtakes), 4.98M files were generated with a volume of $\sim 18 \mathrm{~TB}$.

\section{CONCLUSION}

Parragirls Past, Present provided us with a rare opportunity to collaboratively develop an immersive cinematic experience with a group of women who were highly attuned to the difficultly of witnessing trauma and the pitfalls of documenting memory. Specific challenges we faced in terms of the need to develop radically new ways to shift the didactics of veracity, both in relation to photographic faithfulness and the testimony of trauma.

As mentioned at the outset, this collaboration was undertaken in parallel with a royal commission (Commonwealth of Australia 2015) into the historical sexual abuse of former residents at Parramatta Girls Home. Parragirls' acute direction of this work and our awareness of the conflicted nature of any attempt to document traumatic memory is not only the background of this project but also a response to the limits of the legal system to empower victims. As evidenced in the words spoken by one of the women at the conclusion of the work: 'we can't fix the future if they don't recognise our past'.

The burden of proof also affects the interpretation of heritage for the future, as this culturally and historically significant place is now under threat of major development. As such, Parragirls Past, Present may well be the only comprehensive future archive of the site. The project generated a detailed $3 \mathrm{D}$ reconstruction of the site and selected interior spaces also provide an extensive photographic record, in addition to more than fifty hours of voice and site-based recordings, first-hand accounts and recollections from Parragirls. Finally, this project is a study of alternative approaches to the documentation of contested memory, one that we envisage as opening a significant area for future research in interdisciplinary fields of immersive media, experimental and community arts, cultural heritage, memory and human rights.

\section{ACKNOWLEDGEMENTS}

Parragirls Past, Present: Unlocking memories of institutional 'care', commissioned by The Big
Anxiety: festival of the arts + science + people, Sydney 2017 (Director Jill Bennett). Art Director and Production Design: Volker Kuchelmeister. Sound Design/Editor: Alex Davies. Writer/Editor: Lily Hibberd. Writer/Narrator: Bonney Djuric, Jenny McNally. Narrator: Lynne Edmondson, Paskovski, Gypsie Hayes, Denise Nicholas. Co-producers Jill Bennett, Bonney Djuric, Lily Hibberd. Special thanks to UNSW Epicentre Expanded Perception \& Interaction Centre. For access to the Parramatta Girls Home dataset, please contact the authors.

\section{REFERENCES}

Bartlett, F. C. (1932) Remembering. Cambridge University Press.

BBC (2016) Italy's Invisible Cities.

http://www.bbc.co.uk/programmes/b0881gfb (retrieved 12 February 2018).

Commonwealth of Australia (2015) Royal Commission into Institutional Responses to Child Sexual Abuse, Report of Case Study No.7, Child Sexual Abuse at the Parramatta Training School for Girls and the Institution for Girls in Hay. http://www.childabuseroyalcommission.gov.au/casestudy/f5e0f634-5670-4abf-bdf6-c7d8a58d677f/casestudy-7,-february-2014,-sydney (retrieved 20 March 2018).

Corner, J. (2016) The Thick and the Thin of It. In: Girot, C., and Imhof, D. (eds.), Thinking the Contemporary Landscape. Princeton Architectural Press, pp.117-135.

Girot, C. (2014) Landscape: Beyond the Margins of Vision. In: Deriu, D., Kamvasinou, K. (eds). Emerging Landscapes: Between Production and Representation, Rutledge, London, pp.81-94.

In the Eyes of the Animal (2015). http://iteota.com/ (retrieved 12 February 2018).

Palimpsest (2016)

http://www.interactivearchitecture.org/lab-

projects/palimpsest (retrieved 12 February 2018).

Parragirls Past, Present: Unlocking memories of institutional 'care' (2017) https://parragirlsmovie.com (retrieved 1 March 2018).

PFFP Memory Project (2017)

https://www.pffpmemory.org.au (retrieved 16 March 2018).

ScanLAB (2018) http://scanlabprojects.co.uk (retrieved 12 February 2018).

Schacter, D., and Addis, D. (2007) The cognitive neuroscience of constructive memory: remembering the past and imagining the future. Philosophical Transactions pp.773-786.

Schuemie, M. J., and Charles A. P. G. (1999) Presence: Interacting in VR? Proceedings, Twente Workshop on Language Technology.

So, R. H. Y., Lo, W. T., and Ho, A. T. K. (2001) Effects of Navigation Speed on Motion Sickness Caused by an Immersive Virtual Environment. Human Factors, 43(3), pp.452-461.

Tulving, E. (2002) Episodic Memory: From Mind to Brain. Annual Review of Psychology, 53, pp.5-25. 


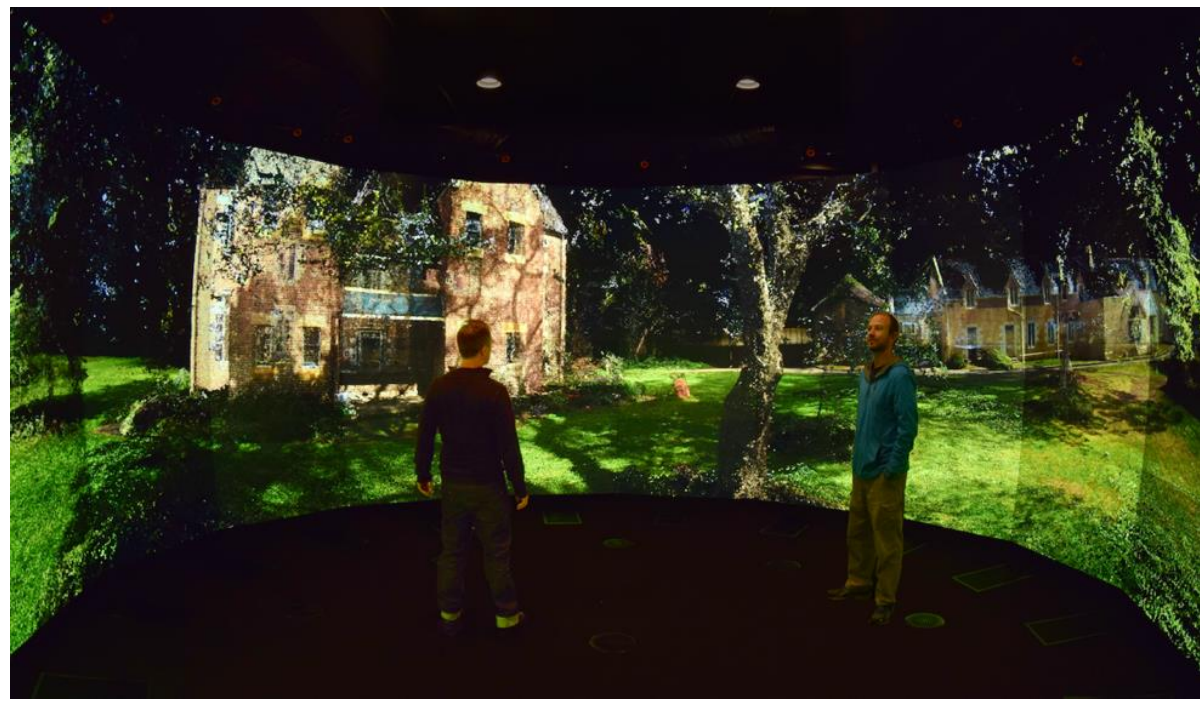

Figure 12: Parragirls Past, Present as presented in EpiCylinder (UNSW Art and Design).
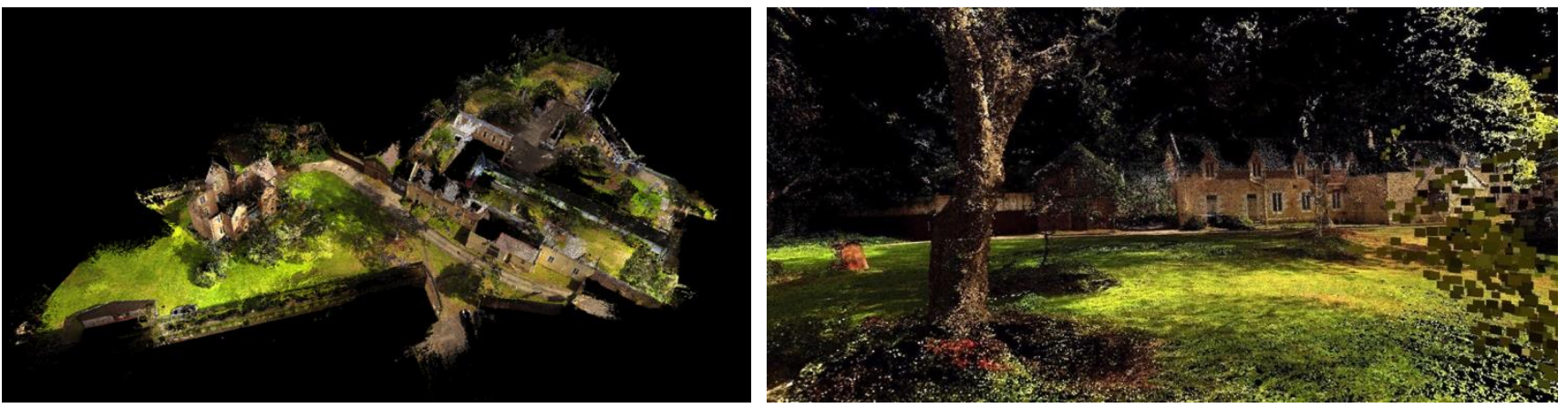

Figure 13, 14: Aerial view point-cloud representation of the site in Parramatta, 1.5ha, 24M points (I). Point-cloud representation close-up (r).
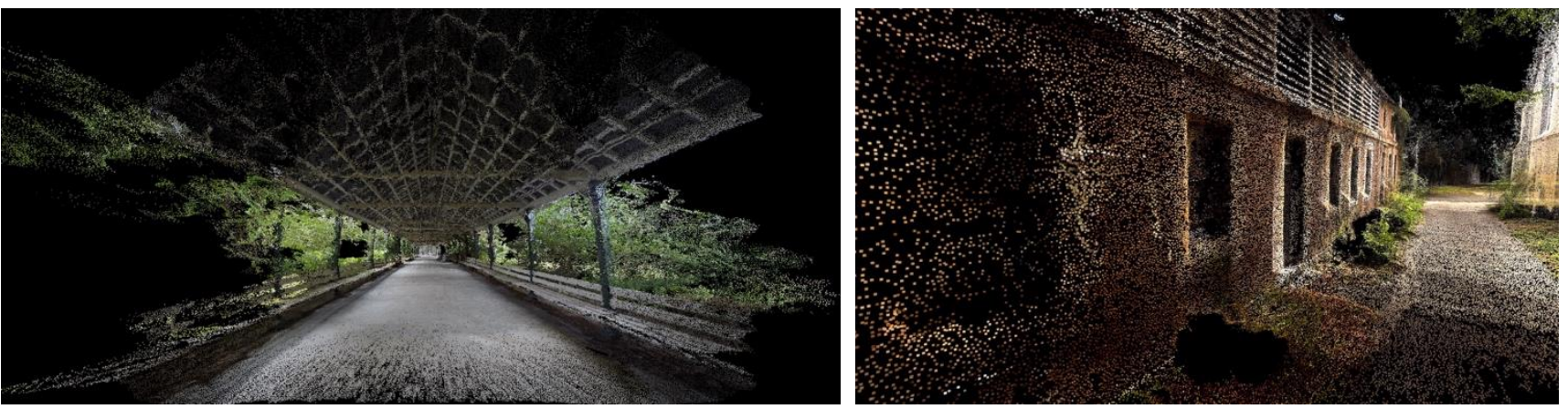

Figure 15, 16: Point-cloud representation of the site in Parramatta close-up.
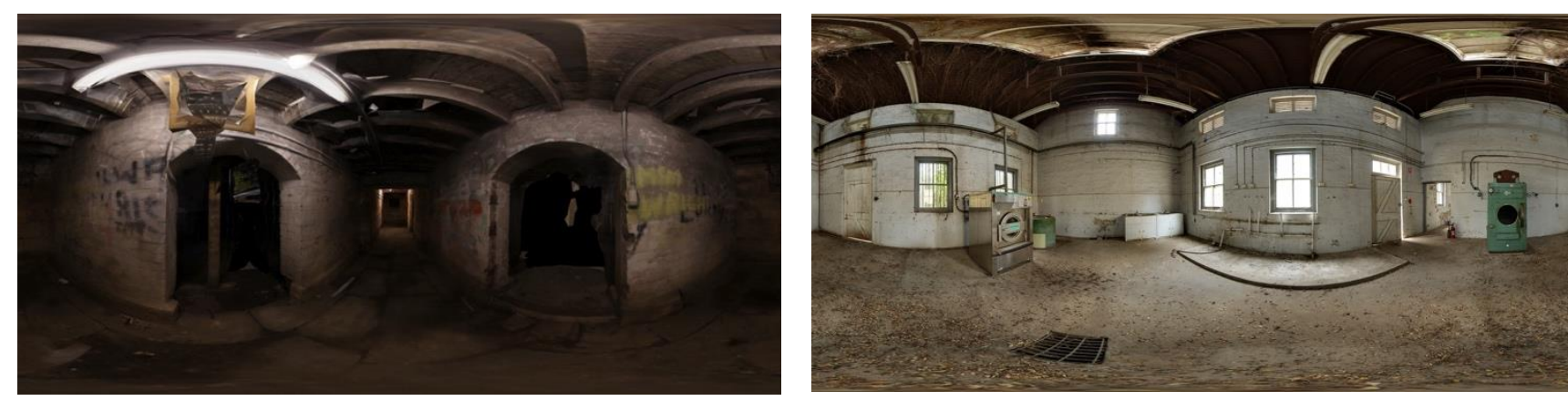

Figure 17, 18: Textured mesh of interior space, spherical 360 view (I). Left eye view of an interior stereoscopic 3D spherical panorama (r). 